\title{
JÓVENES ANTISOCIALES O CON TENDENCIAS SEGÚN LAS PRECEPTORÍAS MEXIQUENSES
}

\section{YOUTH ANTISOCIAL OR WITH TENDENCIES ACCORDING TO MEXIQUENSES PRECEPTORIES}

\author{
Abraham Osorio Ballesteros* \\ Alicia Tinoco-García**
}

RESUMEN

\begin{abstract}
En este artículo se analizan algunas ideas de las autoridades de las preceptorías del Estado de México para explicar la existencia de jóvenes con conductas antisociales entre los sectores populares, con base en una investigación cualitativa realizada en el año 2016. Se destaca que tales autoridades consideran que estos jóvenes se orientan hacia tales conductas, tanto por cambios personales como por situaciones sociales que enfrentan, otorgándole la responsabilidad a ellos y a sus familias, sin considerar los factores estructurales.
\end{abstract}

PALABRAS CLAVE: JÓVENES * CONDUCTAS ANTISOCIALES * AUTORIDADES * ATENCIÓN * SOCIEDAD

\section{ABSTRACT}

Based on a qualitative research of 2016, the article analyzes some ideas by which the authorities of the preceptories of the State of Mexico explain the existence of young people with antisocial behaviors among the popular sectors. He emphasizes that such authorities consider that young people in these sectors are oriented towards such behaviors both by personal changes and by social situations they face, which end up holding them and their families accountable, regardless of structural factors.

KEYWORDS: YOUNG PEOPLE $*$ ANTISOCIAL BEHAVIOR * AUTHORITIES * ATTENTION * SOCIETY

Facultad de Ciencias Políticas y Sociales. Universidad Autónoma del Estado de México. sub_abraham@yahoo.com.mx

** Facultad de Ciencias Políticas y Sociales. Universidad Autónoma del Estado de México. almatiga@gmail.com 


\section{INTRODUCCIÓN}

Aun cuando en México existe una literatura más o menos abundante sobre las instituciones creadas por el Estado para controlar o gestionar las conductas denominadas antisociales de la infancia-juventud ${ }^{1}$ (Sánchez, 2008; Del Castillo, 2006; Azaola, 1991), no existen suficientes trabajos que den cuenta de las existentes fuera de la capital y menos aún, que analicen las posturas manejadas por algunas de sus autoridades.

De hecho, de los estudios denominados "clásicos", quizás solo algunos (Fletes, 1993; Herrera, 2005) toman en cuenta instituciones ubicadas en Guadalajara, Jalisco, Puebla y otras ciudades en donde existen referencias históricas $y$ vigentes del caso. Pero fuera de estos, no abundan los trabajos que indiquen cómo funcionan tales instituciones en otras entidades distintas a la Ciudad de México, lo cual, en el mejor de los casos, ha llevado a que se generalicen ciertos hallazgos de unas latitudes a otras que, aunque son importantes, no son homogéneos y menos aun cuando las políticas federales adaptadas por cada entidad presentan particularidades distintas.

Es en este contexto en el que se inscribe el presente trabajo, el cual tiene por objeto ofrecer una primera interpretación a algunas de las ideas manejadas por las autoridades de las Preceptorías Juveniles Regionales de Reintegración Social del Estado de México para abordar a los jóvenes de los sectores populares considerados en riesgo de cometer conductas antisociales ${ }^{2}$.

$1 \quad$ Por conductas antisociales se entienden normalmente las manifestaciones de conductas que contravienen "las reglas de convivencia, sea[n] [...] [apartadas] de ellas, [o] sean en contradicción con sus preceptos y prohibiciones" (Funciones ejecutivas y conducta antisocial, s.f., p. 1). Por lo tanto, la literatura jurídica nacional incluye dentro de esta, las acciones que infringen las leyes penales 0 reglamentos de policía y buen gobierno, así como, las conductas que afectan la moralidad y seguridad social de las personas y la familia.

2 Las Preceptorías Juveniles Regionales de Reintegración Social del Estado de México (o simplemente Preceptorías como se denominaran en este texto) son instancias públicas dependientes de
Es decir, busca analizar algunas ideas que utilizan tales autoridades para explicar la existencia de jóvenes con conductas antisociales entre los sectores populares $y$ en menor medida, el manejo de ciertas acciones asistenciales en favor de ellos. Sin embargo, no se hace para mostrar las supuestas distancias que guardan con respecto a los propósitos manifiestos de las preceptorías, sino para tratar de identificar las racionalidades políticas en las cuales se basan $y$ por extensión, las cuestiones que destacan de dichos jóvenes.

El supuesto que subyace en el trabajo es que, si bien las autoridades de las preceptorías mexiquenses conciben que la existencia de jóvenes con conductas antisociales entre los sectores populares es resultado de distintos factores, varias de sus ideas enfatizan en cuestiones particulares de ellos y sus familias, que terminan por presentar esta problemática como si fuera un producto de actitudes o dificultades individuales, sin considerar otros factores estructurales.

El texto se divide en tres secciones; en la primera se hace una presentación general sobre el concepto de racionalidades políticas, el cual es retomado del enfoque de la gubernamentalidad para los análisis e interpretaciones subsecuentes $^{3}$. Posteriormente, en la segunda

la Dirección General de Prevención y Readaptación Social (DGPRS) del Estado de México, orientadas a detectar, atender y prevenir la comisión de conductas antisociales entre los sectores juveniles, a través de dos programas denominados de Prevención de la Antisocialidad y de Reintegración para Adolescentes. De acuerdo con su reglamento general, en julio de 2013, existían 24 preceptorías en la entidad -aunque en su página electrónica se mencionan 22- las cuales son atendidas por personal especializado, donde se incluye a un médico, un psicólogo, un trabajador social y un pedagogo quienes son coordinados por un presidente $y$ un secretario de acuerdos; sin embargo, cuando se visitan algunas de estas se percibe que esto no siempre se cumple por los recursos a su disposición, reducidos muchas veces.

3 El enfoque de la gubernamentalidad es un abordaje teórico en constitución derivado esencialmente del concepto de gubernamentalidad introducido por primera vez por Michel Foucault en 1978. Este abordaje ha sido promovido esencialmente por 
sección, se analizan dos conjuntos de ideas utilizados por las autoridades de las preceptorías mexiquenses para explicar la existencia de jóvenes antisociales entre los sectores populares y el manejo de ciertas acciones asistenciales. Por último, en la tercera sección, a manera de conclusiones, se hacen tres reflexiones en torno al texto para indicar lo que dejan entrever las ideas manejadas por las autoridades de las preceptorías en términos de gobierno o control de los sujetos.

\section{BREVE NOTA METODOLÓGICA}

El texto se basa en cuatro entrevistas semiestructuradas $y$ en menor medida, en dos reportes de observaciones participantes realizados por los investigadores y por los miembros de un cuerpo académico, en el marco de una investigación cualitativa sobre las preceptorías mexiquenses auspiciada por la Secretaría de Educación Pública (sEP), a través de un programa orientado a la consolidación de Cuerpos Académicos En Formación (CAEF).

Las entrevistas fueron realizadas entre los meses de marzo y abril del año 2016, siendo aplicadas a dos autoridades (directores) de las preceptorías regionales de Atlacomulco y Metepec, $y$ a un encargado de área de la preceptoría de Toluca, así como, a un ex colaborador de estas dos últimas instituciones, quien ahora labora en la Universidad Autónoma del Estado de México (uaEmex) ${ }^{4}$. Su duración osciló entre los 35 y 90 minutos, llegando en ciertos

seguidores anglosajones de la obra de este filósofo francés como Colin Gordon, Peter Miller, Tomas Lemke, Nikolas Rose, entre otros (Grinberg, 2007). Si bien, cada uno de estos autores apunta a aspectos particulares, todos en general coinciden en la idea de que hay atender la conducción de las conductas en sentido lato para entender cómo se gobierna en el presente, colectiva e individualmente.

4 La preceptoría de Atlacomulco se ubica en el norte de la entidad, mientras que las de Metepec y Toluca en el centro, en las cabeceras municipales del mismo nombre. La primera aborda a jóvenes de 10 municipios, mientras que la segunda y tercera a jóvenes de 14 y 6 municipios, respectivamente, lo que representa el $24 \%$ del total de municipalidades mexiquenses. momentos a adquirir la forma de historias de vida por las cuestiones personales enunciadas por los entrevistados ${ }^{5}$.

El criterio de selección utilizado fue la disponibilidad de las personas, quienes accedieron a colaborar con la investigación después de haberles presentado el guión de la entrevista $y$ de haberlo revisado cuidadosamente. Su experiencia laboral de varios años en las instituciones, los hizo particularmente significativos, ya que pudieron explicar varias situaciones que no se habían previsto, como son algunas diferencias entre ciertos jóvenes abordados por la preceptoría regional de Atlacomulco, debido a sus vínculos con grupos étnicos y los de las preceptorías de Toluca y Metepec, cuyos modos de vida son predominantemente urbanos o semiurbanos, aunque marcados como los anteriores por distintas precariedades o bien, las dificultades operativas que enfrentan las tres preceptorías debido a sus reducidos recursos.

En las entrevistas se trataron varios temas: desde la historia de las preceptorías en la entidad y municipio hasta los problemas que, según los entrevistados, afectan sensiblemente el desempeño de las tareas. Sin embargo, el propósito inicial de este estudio era identificar las opiniones que tenían sobre los jóvenes con conductas antisociales (o denominados en riesgo) y las formas en que los representan, varias de las preguntas fueron direccionadas hacia ello.

Las entrevistas fueron documentadas con grabadoras de voz, para posteriormente transcribirlas $y$ analizarlas con base en la teoría fundamentada desarrollada por Strauss y Corbin (2002), para identificar algunos temas no considerados anteriormente $y$ vincularlos con los planteamientos iniciales. Finalmente, se construyen conjeturas o argumentos sobre el tema de análisis ${ }^{6}$.

$5 \quad$ Con excepción del último entrevistado, quien fue entrevistado en un café de la ciudad de Toluca, el resto ofreció los espacios de sus oficinas para la realización de las entrevistas, ubicadas dentro de las preceptorías.

6 Es importante advertir que el análisis de las entrevistas no siguió estrictamente lo planteado por la teoría fundamentada de Strauss y Corbin (2002) 
Por otro lado, en lo referente a los dos reportes de observaciones participantes, fueron realizados en dos eventos (uno de fútbol $7 y$ otro de ajedrez) organizados por los directivos de varias preceptorías en los municipios de Metepec y Toluca, en dos días del mes de abril de 2016, en el marco de la Semana de la Prevención del Delito auspiciada por la DGPRs del Estado de México.

En tales reportes, se busca representar lo observado y lo conocido en tales actividades, pese a su grado de subjetividad: desde las formas en que se organizan y los problemas que enfrentan las autoridades hasta las personas asistentes, así como, los discursos y las prácticas hacia los padres de familia y jóvenes implicados. No obstante, el interés del estudio estaba más orientado a abordar concepciones o posturas de las autoridades, por lo cual se destacaron ciertos discursos donde se encuentran representadas, los cuales fueron complementados con otros captados en conversaciones informales con autoridades de las preceptorías investigadas.

Los reportes siguieron un plan de acción metodológico previo, el cual consideró primeramente la elaboración de una guía de observación para "saber qué mirar (y escuchar)" en los eventos, posteriormente, la interacción con algunos actores para generar confianza hacia el personal investigador $y$ finalmente, la elaboración de los reportes en los días subsecuentes a los eventos. Después de lo anterior, se hizo una revisión a ambos reportes para identificar cuestiones centrales para el análisis.

Las entrevistas y los reportes de observaciones fueron relevantes en el análisis de este trabajo, ya que permitieron captar discursos e ideas cuyos contenidos son objeto de atención del marco conceptual seleccionado y consecuentemente del artículo.

pues, en lugar de buscar la generación de teoría desde los datos a partir de su codificación (abierta, axial o selectiva) como esta lo sugiere, solo se consideró su idea de abordar línea por línea (o en clave microsociológica) todos los extractos de las entrevistas para descubrir relaciones de ideas $y$ finalmente, con base en el principio conceptual, poder estructurar hipótesis o conjeturas fundamentadas.

\section{REFERENTE CONCEPTUAL PARA EL ANÁLISIS}

Debido a que en las ideas de las autoridades investigadas aparecieron normalmente implicados aspectos relacionados con el control de los jóvenes, se consideró conveniente utilizar para el análisis de estas, el concepto de racionalidades políticas popularizado por Nikolas Rose, Peter Miller, Mitchel Dean y otros autores impulsores del enfoque de la gubernamentalidad. Según Rose y Miller (1992), este concepto hace alusión a los marcos de referencia utilizados por los sujetos para los ejercicios de poder 0 , para ser más precisos, posibilitar las formas de gobierno o control de las conductas no solo de otros, sino de las personas mismas (Grinberg, 2007). Ello debido a que alude tanto a la naturaleza del gobierno como a su práctica, las cuales implican ideas en torno a quién puede gobernar, qué es gobernar y qué o quién puede ser gobernado, mismas que son reproducidas tanto por los sujetos que ejecutan las prácticas de gobierno como por quienes son gobernados (Gordon, 1991).

Las racionalidades políticas incluyen moralidades (en tanto están impregnadas de valoraciones), epistemologías (manejan saberes) $y$ lenguajes (se vuelven pensables a través de ellos), lo cual implica que su análisis puede considerar desde las justificaciones morales utilizadas por autoridades sobre los modos particulares de ejercer el poder, hasta las nociones sobre las formas apropiadas de la política y la distribución de tareas entre las diferentes entidades, ya sean seculares, espirituales, militares, familiares o pedagógicas (De Marinis, 1999).

De acuerdo con De Marinis (1999), el concepto de racionalidades políticas puede prestarse a distintas interpretaciones, pues se trata de una noción que atraviesa el pensamiento filosófico y social moderno; no obstante, como en el enfoque de la gubernamentalidad no contempla la carga normativa $y$ se inclina hacia la utilidad práctica, aquí se le considerará como un concepto de valor empírico, que tiene "un significado meramente relativo a determinadas prácticas históricas" (p. 14); lo que significa que cada instancia de gobierno puede manejar diferentes racionalidades (en función de sus propósitos manifiestos y no manifiestos) 
$y$ las de sus actores, sin que esto implique una desconexión entre las entidades, pues también comparten posturas de racionalidades políticas estructurales o mayores, cuyos rasgos pueden apreciarse incluso entre las nociones de los sujetos. Por ejemplo, actualmente, las reducidas acciones asistenciales en favor de población infantil y adolescente de las calles en la ciudad de México, no pueden entenderse sin considerar la racionalidad política neoliberal en boga que, bajo el supuesto de reconocer las capacidades de los sujetos, alienta un apoyo condicionado. Pero tampoco puede entenderse sin considerar ciertas ideas relacionadas con la racionalidad política de la doctrina irregular - antecesora de la neoliberal - la cual alienta una gestión tutelar de los niños de la calle por parte del Estado (Rose, 2007), así sea de manera autoritaria o arbitraria para evitar su transformación en un problema mayor.

Finalmente, el concepto de racionalidades políticas está normalmente asociado con el de tecnologías de gobierno, el cual también fue acuñado dentro del enfoque de la gubernamentalidad. Este último concepto hace referencia a "los procedimientos prácticos por los cuales se inscribe el ejercicio del poder, la autoridad $y$ el dominio" (Graizer, 2007, p. 9), es decir, a las prácticas cotidianas por medio de las cuales se logra gobernar o manejar a las personas (Dean, 1999). De manera tal que, como dice De Marinis (1999), un análisis de estas tecnologías remite directamente a un enfoque micro-físico del poder, toda vez que:

... no se trata de grandes esquemas políticos de conjunto, ni de modificaciones ideológicas, ni racionalizaciones ni sistematizaciones, sino de mecanismos prácticos y reales, locales $y$ aparentemente nimios, a través de los cuales los diversos tipos de autoridades pretenden conformar, analizar, guiar [o] instrumentalizar las [...] aspiraciones, pensamientos $y$ acciones de los otros, a los efectos de lograr los fines que ellas consideran deseables (p. 15).

No obstante, es importante recalcar que para efectos de este trabajo no se retomará, ya que se consideró que la vinculación entre las racionalidades y las tecnologías de gobierno es fundamental para una comprensión holística de estas, como lo plantean algunos autores, aquí únicamente se orientará a mostrar cómo las prácticas de gobierno toman forma en términos esquemáticos o son conceptuadas por las autoridades de las preceptorías mexiquenses $y$ por lo tanto, cómo imaginan a los sujetos atendidos o abordados. Aspectos necesarios para manejar una visión crítica de tales instituciones pero también para problematizar su funcionamiento.

\section{LOS JÓVENES CON CONDUCTAS ANTISOCIALES O EN RIESGO DE TENERLAS SEGÚN LAS AUTORIDADES DE LAS PRECEPTORÍAS MEXIQUENSES}

Del conjunto de datos recabados de las autoridades preceptoras mexiquenses, se identificó la siguiente postura entre ellas: que los jóvenes de los sectores populares se orientan (o pueden orientarse) hacia la comisión de conductas antisociales más que los jóvenes de otros sectores, tanto por una serie de ajustes personales como por varias situaciones sociales que enfrentan, los cuales, aunque no son particulares, les afecta principalmente a sus personas e incrementa su proclividad hacia ello.

A partir de lo anterior, las autoridades indican que las conductas antisociales entre los jóvenes de los sectores populares son resultado de diferentes factores, los cuales destacan esencialmente actitudes o problemas particulares de ellos y sus familias, que terminan por responsabilizarlos y desconocer otros "aspectos estructurales"7.

$7 \quad$ Por aspectos, cuestiones o factores estructurales se entenderá en este trabajo a las condiciones de pobreza, exclusión y desigualdad, las cuales pueden ser atendidos por el Estado. Esta acepción se fundamenta en la propuesta de Johan Galtung (s.f.), particularmente en su noción de violencia estructural utilizada en estudios de violencia en América Latina y varias partes del mundo, aunque de una forma particular para considerar el papel del Estado como responsable de dichos aspectos aun cuando racionalidades neoliberales traten de no considerarlo. 
LOS CAMBIOS PERSONALES COMO

ALENTADORES DE CONDUCTAS ANTISOCIALES ENTRE LOS JÓVENES

En referente a los ajustes personales de los jóvenes, entendidos como cambios físicos y de actitudes que los llevan a asumir distintas posturas en torno a algo, las autoridades de las preceptorías mexiquenses consideran que algunos cambios pueden inducirlos hacia conductas desaprobadas, aunque entre los más importantes se destacan: la inconformidad con lo poseído, la desatención que sienten de sus padres o tutores, la apelación a sus derechos y el riesgo que normalmente buscan experimentar.

En el caso concreto de la inconformidad con lo poseído, las autoridades consideran que los orienta (o puede orientarlos) hacia el cometimiento de conductas antisociales debido a los sentimientos de intranquilidad $y$ ansiedad que les genera comparar sus precariedades con "lo que otros tienen" y cuando conciben que pueden mejorarlas llevando a cabo acciones fuera de lo permitido. Es decir, cuando identifican las diferencias marcadas que existen entre lo poseído por ellos y lo de otros, lo que se puede mejorar llevando a cabo conductas desaprobadas. Tal como lo manifestaba el encargado de área de la preceptoría de Toluca en una conversación informal: "la insatisfacción con lo tenido les genera ansiedad para hacer cosas fuera de lugar, para orientarse hacia otras cosas" (C. González, comunicación personal, marzo de 2016) ${ }^{8}$ o como lo señalaba el preceptor de Atlacomulco, recordando la reflexión que hacen varios jóvenes en proceso de reintegración antes de cometer nuevamente un acto antisocial y en donde aparece una suerte de comparación: "Si yo ya estuve ahí [en una institución de reintegración], pues no me importa que me vuelvan otra vez a agarrar para [...] por lo menos [...] seguir disfrutando [de ciertas cosas] [...]" (A. Nolasco, comunicación personal, abril de 2016).

Esto no significa, dicen las autoridades, que todos los jóvenes de los sectores populares se orienten hacia ello pues, si bien existe algún grado de inconformidad debido sus condiciones

8 Por respeto a las autoridades entrevistadas, se han modificado sus nombres. de vida, la mayoría desarrolla una tolerancia positiva hacia las precariedades, la cual los lleva a decir, según una autoridad: "mmm ¡No!, No le entramos" (R. Rodríguez, comunicación personal, abril de 2016). Según las autoridades, así como existen personas con distintos grados de tolerancia hacia la violencia, existen sujetos con distintos niveles de tolerancia hacia las precariedades económicas.

A partir de lo anterior, se puede inferir entonces una especie de racionalidad psicológica en el tema por parte de los preceptores. Esta racionalidad que utiliza saberes (De Marinis, 1999) de la elección racional y en menor medida, de la personalidad. Como lo indican ciertas teorías, algunos jóvenes de los sectores populares pueden llevar a cabo acciones antisociales por el simple hecho de convenir a sus intereses y no saber controlar su frustración económica, creando con ello la imagen de sujetos singulares, entre racionales y desadaptados.

Lo anterior no es un asunto menor, puesto que las autoridades los responsabilizan directamente de dichas acciones, $y$ dejan de reconocer a la pobreza $y$ otros aspectos como factores estructurales de fondo que subyacen debajo de sus decisiones $y$ al Estado como garante, tal y como lo plantea la racionalidad política neoliberal vigente, la cual considera la existencia de grupos diferenciados, sojuzgando a los denominados "desordenados" (jóvenes con diferente conducta que los demás), sin considerar las condiciones macrosociales que les afectan en el marco de un Estado "mínimo" (Hener $y$ Niszt, s.f.).

En una línea cercana a la anterior, se ubica - aunque con sus diferencias- la explicación de las autoridades en el tema de la desatención paternal. De acuerdo a las entrevistas, la desatención de este tipo puede conducir a los jóvenes de los sectores populares a cometer conductas antisociales debido al resentimiento personal que llegan a generar en contra de sus padres u otras figuras de autoridad, el cual posteriormente y bajo ciertas condiciones, puede llevar en acciones no toleradas. Una de las frases más claras que evidencia esta postura, aunque planteada para otro caso, es la mencionada por uno de los preceptores: "sabemos que cuando 
los jóvenes tienen desatención de sus padres van adquiriendo resentimiento $y$ desafección hacia ellos lo cual al final de cuentas puede orillarlos a hacer cosas fuera de la ley, por lo que en estos torneos buscamos que también estén sus padres" (M. Estrada, comunicación personal, abril de 2016).

Según lo señalado por las autoridades, ese resentimiento en muchos jóvenes solo queda en reproches hacia sus padres o tutores, incluso en desapego hacia ellos, pero en otros casos, termina en desacato de las normas, pues con el paso del tiempo "se va[n] mentalizando a tener problemas" (A. Nolasco, comunicación personal, abril de 2016) hasta que finalmente los tienen.

Si bien, en esta explicación de las autoridades no se advierte una implicación de saberes racionalistas y de la personalidad para clarificar la emergencia de conductas antisociales, sí se identifica saberes, aunque no para argumentar dichas conductas como consecuencia de una frustración, sino como resultado de un resentimiento individual. En efecto, en esta explicación se deja entrever una racionalidad psicológica de que los jóvenes de los sectores populares pueden llevar a cabo actividades antisociales por el hecho de guardar algún rencor hacia sus padres derivado del abandono (o de la percepción de abandono).

El problema de dicha argumentación radica en que, sin pretenderlo, termina nuevamente por atomizar la temática de las conductas antisociales considerándolas, en este caso, como consecuencias de sentimientos personales de los jóvenes y poniendo como responsables directos a sus padres o tutores.

Por otro lado, en lo referente a la apelación de los derechos de los jóvenes, las autoridades de las preceptorías conciben que este factor incrementa la proclividad de cometer conductas nocivas entre esta población, por el hecho de generarles una especie de confianza excesiva de llevar a cabo distintas acciones, incluyendo las nocivas, sin preocuparse en las consecuencias. Un ejemplo representativo de esta postura aunque referido para jóvenes reincidentes, es el señalado por el excolaborador de la preceptoría de Toluca, quien manifiesta la supuesta confianza que sentían algunos jóvenes después de cometer alguna falta: "Entonces estábamos ahí en una oficina [con los tres jóvenes mencionados y dicen] [...]: no se preocupen, [...] no nos puede hacer ni madres, somos menores de edad, [tenemos derechos], ustedes no la hagan de pedo y no vayan a rajar putos" (R. Rodríguez, comunicación personal, abril de 2016).

Lo anterior como consecuencia del conocimiento que han adquirido de los beneficios que llegan a obtener por aludir a sus derechos, el cual aunque puede ser general e impreciso está basado en su experiencia personal. Esto los lleva a cometer actividades ilícitas no solo en las calles o en las escuelas donde se desenvuelven, sino también al interior de sus hogares donde incluso llegan a violentar a algún familiar, conscientes de que no tendrán consecuencias legales. Por lo cual, las autoridades terminan planteando una atención judicial hacia los jóvenes como si fueran adultos, como una manera de controlarlos: "[...] hay chavos que ya conocen la ley, ya saben qué es lo bueno y lo malo y [se requiere] [...] que sean tratados como adultos. [Sobre todo porque] Se ha visto tanta maldad [entre ellos últimamente)" (C. González, comunicación personal, marzo de 2016).

Haciendo un análisis general a esta explicación de las autoridades respecto a los derechos como un elemento que incrementa la proclividad de cometer conductas antisociales, se infiere que esta se sostiene en esquemas de referencias utilitarias $\mathrm{u}$ oportunistas que ubican a esta población como conocedores de la temática pero sobre todo estratégicos. Desde la lógica de las autoridades, estos jóvenes manejan el discurso de los derechos humanos para obtener beneficios $y$ de esta manera, utilizarlos a su favor cuando comenten algún acto antisocial e incluso, en momentos de sojuzgamiento hacia sus personas.

Este hecho es relevante ya que se refuerzan las percepciones negativas hacia estos jóvenes en el imaginario social y consecuentemente, permite la posibilidad de manejar acciones de control más severas hacia ellos (tratarlos judicialmente como adultos), cuya lógica supone ser 
menos tolerantes con sus acciones para evitar su escalada antisocial o delincuencial. Asimismo, esto alude a parte de la doctrina tutelar vigente hace varias décadas en México $-y$ metamorfoseada en la actualidad con la asunción de teorías criminalísticas - según la cual, la infancia-juventud de los sectores populares tiende a sacar más beneficios de ciertas situaciones (como en este caso serían sus derechos) que otros sectores, por lo que es necesario ser más severos con esta población.

Respecto a la supuesta tendencia hacia el riesgo de estos jóvenes, vale indicar que las autoridades consideran que esto incentiva a las conductas antisociales, por el hecho de inducirlos al peligro y a la experimentación; ejemplos característicos de lo anterior son las ingestiones de bebidas alcohólicas o consumo de drogas, utilizadas muchas veces para explorar o sentir cosas.

Esta tendencia es visualizada por las autoridades como parte de una naturaleza juvenil o de un proceso de desarrollo, no obstante, es considerada como relevante entre los jóvenes de los sectores populares por las costumbres que aprenden en sus contextos. Una de estas costumbres es la actitud permisiva de algunos de sus padres quienes, por comodidad o desinterés, les toleran la realización de actividades nocivas, aun cuando estas "pueden generarles ciertos problemas" o naturalizarlas, como lo señala uno de los preceptores entrevistados:

[...] [hay] [...] papás [...] [que dicen] sabes qué, yo no quiero que tomes en la calle [...] [mejor vente] aquí en la casa y ese es un error [...] [porque] hay niños que tienen 8 años y ya [...] [aprendieron a] distinguir [...] cuál es el whiskey, cuál es el tequila y cuál es el de mejor calidad, entonces [...] por eso hay muchos niños que [...] son alcohólicos [...] (A. Nolasco, comunicación personal, abril de 2016).

Según se indica en los comentarios de las autoridades, cuando los padres son permisivos con los jóvenes, permiten que estos no consideren las normas sociales y su tendencia hacia el riesgo se multiplique. Otra de las costumbres destacadas por las autoridades es el "libertinaje juvenil", el cual induce a varios jóvenes a cometer acciones peligrosas o al límite por la búsqueda del sentimiento de libertad. Tales son los casos de los varones que, por quedar bien con sus amigos, deciden participar en actos incorrectos o de riesgo; además existe el caso de mujeres que, para validar sus sentimientos a sus parejas, sentir adrenalina o ser partícipes de algo, se involucran en situaciones riesgosas planificadas por los hombres sin considerar las consecuencias. Sobre estos últimos casos, uno de los preceptores mencionó el caso de una mujer partícipe en robos de autopartes, debido a que su pareja lo llevaba a cabo, mientras que en otro caso, describió el proceder irreflexivo de algunas mujeres que participaban en acciones delictivas por querer experimentar algo:

[...] un[a] adolescente mujer, puede cometer un delito como un adolescente hombre, [...] pero las mujeres [...] no lo organizan, o sea no lo planean, o sea ellas, nos decían en la película del famoso "Borras" [....] [solamente] se [...] [van] al aventón [...] (A. Nolasco, comunicación personal, abril de 2016).

Sin ahondar en el análisis, esta argumentación describe la tendencia hacia el riesgo como incentivadora de conductas antisociales, es un marco de referencia (o racionalidad) de las autoridades basado en posturas socializantes, cuyas ideas aparecen marcadas de valoraciones. Según las autoridades, este marco de referencia permite visualizar una especie de naturaleza juvenil que pondría a los jóvenes de estos sectores en una ruta de riesgo, potencializada por una serie de costumbres que implican una transgresión a los límites de lo tolerable e incentiva el desacato a las normas. De manera que los términos utilizados para definir tales costumbres aluden a excesos: permisividad como más allá de lo permitido y libertinaje como una libertad excesiva o abusiva.

Más allá del cumplimiento de este marco de referencia, lo que genera una imagen "desfavorable" de los actores involucrados (jóvenes y familiares), pues los presenta como personas irresponsables (como en el caso de los padres que permiten el consumo 
de bebidas alcohólicas a los jóvenes en sus casas), con excesos (como el caso de los jóvenes que participan en actos incorrectos para ser aceptados en su grupo de pares) o con altas dosis de inconsciencia (como es el caso de las jóvenes que experimentan emociones participando en actos delictivos).

\section{LAS SITUACIONES SOCIALES QUE PONEN EN RIESGO A LOS JÓVENES DE LOS SECTORES POPULARES}

Aun cuando las autoridades investigadas consideran que los cambios personales en los jóvenes de los sectores populares tienen un papel central en su orientación hacia las conductas antisociales, también consideran que esto se fortalece por ciertas situaciones sociales que experimentan en su entorno. Una de estas situaciones es la existencia de conductas disfuncionales en sus hogares por parte de algún miembro de su familia, las cuales son representadas como acciones atípicas y anormales que pueden incentivar conductas antisociales entre sus miembros más jóvenes, debido a que son normalizadas desde su infancia y reproducidas durante su proceso formativo. Una de las personas representantes de las autoridades de la preceptoría de Atlacomulco mencionaba el ejemplo de un joven sicario y de la influencia negativa que tuvo su familia en relación con sus actividades delictivas.

... [el] famoso Ponchis, o sea [...] el [...] sicario de [...] los narcotraficantes [...] comenzó a cometer esos delitos, porque [...] siempre fue un menor en abandono, o sea, no le ponían atención, no tenía la figura paterna, nada más la que tenía era la materna. Entonces él veía, como vivía en el campo allá en Puebla, [...] que su abuelita mataba a los pollos, pero ella lo hacía con la finalidad de comer, de darles de comer; pero él pensaba que su abuelita los mataba porque ella quería matar, entonces él se fue mentalizando en eso, y él para matar a las personas, [...] mataba perros, mataba gallinas, mataba pollos, conejos, todo eso, o sea siempre los degollaba, entonces ahí fue la cuestión que él utilizó para [hacer lo que hacía] [...] cuando ya lo contrataron [...] (A. Nolasco, comunicación personal, abril de 2016).

Independientemente de que el ejemplo enunciado remite a un caso extremo, la argumentación deja entrever una racionalidad causal en el tema. Una racionalidad basada en cierto saber sociológico de corte funcionalista que concibe que las personas adultas influyen ampliamente en la formación conductual de los jóvenes, en tanto les inculcan desde la infancia, esquemas de obrar y pensar para actuar en sociedad. De modo que si en un hogar existen prácticas o comportamientos disfuncionales por parte de algún miembro es prácticamente un hecho que estos se arraiguen y se naturalicen como parte de la vida cotidiana, como habría pasado en el caso del joven denominado "Ponchis", quien habría aprendido a matar por una suerte de socialización perniciosa de su abuela, que le habría impedido distinguir entre matar animales $y$ matar personas.

De acuerdo con las autoridades de las preceptorías, existe una especie de correlación positiva entre la variable adultos y la variable conductas juveniles, en la cual la segunda sea prácticamente considerada consecuencia de la primera, que en el fondo termina por responsabilizar a los padres de familia. A pesar de lo anterior, las autoridades están conscientes, por un lado, que no en todos los hogares donde hay adultos con problemas de conductas existen hijos con esas mismas conductas; por otro, que si bien los adultos tienen un peso importante en la formación conductual de los jóvenes, no son los únicos ni los determinantes, pues también influyen otros factores sociales como la escuela, las relaciones de pares y los aspectos estructurales, los cuales al combinarse con las posturas de estos jóvenes, pueden generarles distintas conductas en la sociedad.

Otra de las situaciones sociales que vive esta población y que según las autoridades, incentiva la adhesión a conductas antisociales es el manejo de Internet, el cual se ha hecho accesible debido a la cantidad de negocios particulares (denominados cafés internet) establecidos dentro 
de sus colonias o comunidades. Asimismo, al igual que la televisión, el Internet ha posibilitado que estos jóvenes sientan mayor atracción a las conductas delictivas o antisociales, al estar expuestos a distintas escenas de violencia y acciones nocivas. Así lo argumentaba una de las autoridades:

[...]lo de ahorita [...] es [...] internet [...] porque [allí] ellos ven [...] de qué forma pueden llamar la atención [...] para cometer un delito [...] porque [como en ] [...] la televisión, [y] sus famosas [...] telenovelas, [...] ahí los inyectan mucho de la drogadicción, o sea a la violencia, entonces ellos ven eso y lo quieren llevar a la práctica [...] esos son [...] elementos que incitan a un adolescente a cometer ese tipo de conductas para que ellos llamen la atención y para que sus papás también los empiecen a tomar en cuenta (A. Nolasco, comunicación personal, abril de 2016).

Esta visión muestra preocupación sobre la población joven, en tanto considera que el uso "no propio" del Internet puede encauzar hacia conductas antisociales, sin embargo, es una posición desvalorativa de la persona, puesto que presenta a los jóvenes como sujetos incapaces de filtrar la información de este medio de comunicación, por lo tanto, surge la necesidad de ser intervenidos por las autoridades, tal como se realizaba en otras épocas hacia las personas de escasos recursos (Gaytán, 2006). Esto llama la atención pues contrasta con la imagen de sujetos estratégicos (casi manipuladores) presentada por las autoridades cuando se refieren al uso que hacen los jóvenes de sus derechos, aunque comprensible si se considera que paradojas como estas se enuncian en varios momentos desde las instituciones de distintos niveles, como cuando se dicen que es necesario bajar la edad penal para imputarles cosas, pero al mismo tiempo, se les niega el derecho a votar hasta no cumplir su mayoría de edad.

Asimismo, esta visión juzga a las familias porque alude que los jóvenes retoman posturas inadecuadas de Internet con el objetivo de llamar la atención de sus padres. Más aun cuando las autoridades apelan a una suerte de idea valoradora de la familia cercana a lo que Makrinioti y Qvortup denominan como "familiarización" (Gaytán, 2006), la cual considera una fusión "natural" de la infancia-adolescencia con la institución familiar $y$ de manera particular con sus padres, hasta el punto de indicar que cuando hay un abandono por parte de estos últimos, los miembros más jóvenes lo resienten.

Finalmente, una tercera situación social considerada por las autoridades es la socialización de los jóvenes con sus pares o sus amigos. Las relaciones con otros jóvenes o personas de sus barrios pueden ser detonantes de conductas antisociales, debido a los comportamientos nocivos de estos últimos y que son reproducidos por los jóvenes por resultarles llamativos o novedosos. Así lo ilustra uno de los preceptores entrevistados para referirse al caso de los jóvenes influyentes:

[...] hay muchos adolescentes que [...] [por ejemplo van a Querétaro] [...] a trabajar allá, forman ese tipo de patrones de conducta [...] y los traen para acá [en Atlacomulco] [...] Y [esto también ocurre con los] de Ixtlahuaca, [allí] [...] los que se van a la ciudad, [...] en el Distrito Federal pues [...] van a aprender [...] [...] a fumar, [...] a tomar marihuana o la cocaína y [a] [...] [vestirse] de esta forma [estoperoles, camisas negaras, botas] [...] y todas esas costumbres los traen [...] a las zonas de por acá [y eso llama mucho la atención de los jóvenes de la zona] [...](A. Nolasco, comunicación personal, abril de 2016).

Sin ser determinantes en estas aseveraciones se puede intuir que bajo esta argumentación subyace una noción de la subcultura de la violencia, según la cual los grupos de pares influyen en las conductas antisociales de otros cuando socializan distintas conductas de este tipo, las estandarizan como comportamientos $y$ las transmiten en sus relaciones cotidianas (Tello y Garza, 2004). Si bien, dicha noción tiene seguidores entre distintas disciplinas, resulta criticable en este estudio, pues además de apelar a un pasado armónico comunitario 
donde estarían instalados los jóvenes locales con sus tradiciones, valora negativamente a otros grupos de jóvenes con experiencias en otros contextos por el simple hecho de traer consigo otras ideas, prácticas y vocabularios. Es decir, aque al ocurrir aquello nuevamente, se considera a las conductas antisociales como resultado de actos individuales, aunque en este caso sea producto de la influencia de terceros calificados negativamente, sin considerar el impacto de los aspectos estructurales.

\section{LAS ACCIONES QUE ALIENTAN LAS AUTORIDADES DE LAS PRECEPTORÍAS PARA ATENDER A LOS JÓVENES CON CONDUCTAS O CON TENDENCIAS ANTISOCIALES}

Como respuesta a lo anterior referente a los factores desencadenantes de las conductas antisociales en esta población, las autoridades de las preceptorías alientan el manejo de acciones orientadas hacia la corrección de los aspectos psíquicos y familiares, que les permita encaminarse hacia lo solicitado por la sociedad, entendiéndolo como su ocupación en algo productivo. Dentro de estas acciones, las más enunciadas por las autoridades son: las pláticas sensibilizadoras, los talleres y los torneos, las cuales, al centrarse en los sujetos, terminan por no considerar las cuestiones estructurales que les afectan.

Por lo referente a las pláticas sensibilizadoras, las autoridades señalan que se tratan de charlas dirigidas a concientizar a los jóvenes involucrados $y$ a sus padres o tutores. En estas se explican que son las conductas antisociales, sus manifestaciones $y$ sus consecuencias en términos sociales y judiciales. Según las preceptorías, se busca generar una asimilación de las normas, valores y hábitos compartidos para identificar las conductas problemáticas para su posterior modificación. Esto de manera paralela con la obligación de los jóvenes de acatar las normas sociales, así como, los padres y tutores tienen el compromiso moral y familiar de responsabilizarse de la formación ordenada de los jóvenes, pues en caso contrario, la atención no sirve de nada. Más aún cuando buscan apoyar a jóvenes con antecedentes delictivos, ya que en dichos casos: "[...] la tarea es [...] tanto familiar como individual, o tanto individual como grupal [...]" (M. Estrada, comunicación personal, abril de 2016).

Si bien, no se tuvo la posibilidad de presenciar una charla de este tipo, entre las autoridades de las preceptorías y los jóvenes con sus padres, en las entrevistas se dejo entrever sesiones individuales y grupales con temas para tratar de forma individual y en conjunto, lo cual supone una idea arraigada de que ambos son sujetos singulares pero complementarios; singulares porque poseen una naturaleza psíquica individualizada, aunque un tanto desorientada; y complementarios porque para la atención del problema deben involucrarse ambos.

En esta misma lógica se ubica la idea manejada por estas autoridades para acabar con el círculo de la violencia que, según estas, varios hogares de los sectores populares sufren. De acuerdo con las autoridades, para acabar con este es necesario que cada uno de los miembros de la familia haga su parte, disminuyendo sus posturas violentas $y$ alentando sus tratos respetuosos, como toda familia "normal" lo haría.

A partir lo anterior, se puede inferir que para las autoridades, las pláticas sensibilizadoras son visualizadas como acciones recuperadoras, restablecedoras o fortalecedoras, según sea el caso, de la conciencia familiar de los jóvenes $y$ sus padres, quienes la habrían perdido (o estarían en riesgo de perderla) por desatender las normas sociales $y$ apelar a sus intereses personales antes que a los sociales; es decir, son visualizadas como acciones destinadas a recuperar o fortalecer los valores familiares. Esto es representativo de una racionalidad gubernamental de corte neoliberal que busca dirigir el comportamiento de los actores desde el gobierno de sus conciencias, como diría Foucault, pero al hacerlo igualmente desconsidera aspectos macros que sobrepasan las voluntades de los sujetos, sobre todo porque pretende destacar los elementos subjetivos de esta población.

Por otro lado, los talleres como los de elaboración de gelatina, pintura y teatro, están institucionalizados en al menos una de las instituciones conocidas desde hace varios años y reciben a varios jóvenes en horarios específicos. 
Asimismo, funcionan dentro de sus instalaciones con dos objetivos principales: el primero es enseñar el manejar su tiempo, el cual por el contexto en donde se desarrollan, puede estar siendo subutilizado o dirigido hacia otras actividades; el segundo es para crear habilidades productivas que muchos carecen o desconocen por no tener los recursos necesarios para identificarlas.

De acuerdo a las autoridades, quienes han participado en los talleres han logrado manejar su tiempo de ocio a través del aprendizaje de oficios, tal como afirma uno de los preceptores, en el caso de algunos niños que después de haberse integrado a los talleres, aprendieron a realizar cosas manuales y "[....] sacar un provecho [...] [para ellos y sus familias]" (M. Estrada, comunicación personal, abril de 2016). Lo anterior también se aplica para los jóvenes sin experiencia previa en los talleres o desconocen sus habilidades, pues — según las autoridadestan pronto empiezan los cursos y los conocen, quedan emocionados a tal grado de que "hasta los chavos lo piden [nuevamente]" (M. Estrada, comunicación personal, abril de 2016).

Las autoridades suponen que pueden mantener ocupada a esta población juvenil y evitar que se incline a realizar actividades problemáticas, principalmente porque se considera que no saben utilizar su tiempo libre o no conocen sus capacidades para realizar otras labores. En este sentido, dichos talleres son visualizados como mecanismos para que jóvenes pueden aprenden a gestionar su tiempo y adquirir destrezas, como un gobierno de sus personas. Un gobierno que busca la mejoría de ellas "[...] para contribuir, como [potenciales] trabajadores [o ciudadanos], a la mejoría [de la sociedad]" (Gajardo y Milos, s.f., p. 176), aun cuando esto implique no considerar otros aspectos estructurales. Similar a lo que buscaban las escuelas de artes $y$ oficios creadas en el siglo xix —aunque con sus particularidades-, las cuales buscaban "convertir en personas útiles a los menesterosos y desocupados" (González, 2012, p. 149), no obstante, no se consideraban las desigualdades.

Finalmente, en los torneos (particularmente de ajedrez y fútbol) participaban los jóvenes, sus familiares, las autoridades $y$ otros actores, con el objetivo de ayudar a esta población a su formación o reformación, mediante la socialización, la competencia sana y los hábitos hacia las actividades físicas y/o mentales.

De acuerdo con las autoridades, la socialización se presenta cuando los jóvenes aprenden a convivir en grupo y limar asperezas; la competencia sana se da a través del orden y el respeto hacia el contrincante para lograr ciertos fines (o premios); y los hábitos se desarrollan con la práctica continua de las actividades.

Durante la asistencia de los investigadores a estos torneos, se identificó que efectivamente respondían a esos propósitos e implicaban otros de carácter psicológico, como la disminución del "estrés y ansiedad [...] potenciando su autoestima y autocontrol emocional" (Jiménez y Durán, 2005, p. 15), lo relevante en este caso es que las autoridades buscan maneras de gobernar las conductas de los jóvenes desde la diversión antes que la imposición. Esto en un reflejo de una nueva racionalidad gubernamental cuyas argumentaciones plantean, entre otras cosas, la creación de espacios para los sujetos, en los cuales puedan expresar sus habilidades físicas o mentales con miras a gobernarlos desde un no-gobierno o sin parecer que se les gobierna.

Sin embargo, estos torneos desconsideran aspectos no individuales o estructurales que influyen en las conductas antisociales, pues parten del supuesto de que cuando los jóvenes participan en estos, mejoran sensiblemente sus conductas, lo cual no necesariamente ocurre, pues algunos se involucran solamente para evitar problemas con las autoridades.

Estas cuestiones parecen no ser una preocupación para las autoridades de las preceptorías, quienes piensan que si ciertos sujetos se abordan de una manera, el resto de los jóvenes también puede ser trabajados así (Douglas, 1989).

CONCLUSIÓN: REFLEXIONES SOBRE LAS IDEAS DE LAS AUTORIDADES EN CLAVE DE CONTROL O GOBIERNO

Una primera reflexión sobre las ideas manifestadas por las autoridades respecto al papel que juegan la insatisfacción, el resentimiento, el oportunismo y el riesgo en la 
emergencia de conductas antisociales entre los jóvenes de los sectores populares, es que en estas subyacen principalmente racionalidades de tipo psicológica y utilitaria (o una mezcla de ambas), las cuales ponen énfasis en las reacciones y acciones de los sujetos. Lo anterior, si bien ayuda a entender cómo pueden influir cuestiones personales o de utilidad en la aparición de este tipo de conductas, tiene el problema de tratar a los jóvenes y sus familias de manera atomística, casi aislada, olvidando los factores estructurales que también influyen en ellos.

Esto, en clave de gobierno, lleva a dos cosas: en primer lugar, a manifestar posturas críticas y hasta desvalorativas de estos actores a quienes se les ubica como responsables directos de dichas conductas, sea porque no se logran conformar con lo tenido, no logran minimizar su resentimiento hacia sus padres, o no logran contener su oportunismo, en el caso de los jóvenes, o bien, no se dedican a cumplir sus tareas con sus hijos, en el caso de los padres. En segundo lugar, implica que la atención sea de manera atomística bajo la idea de socializarlos, transformarlos o formarlos como lo requiere la sociedad, pues se considera que si sus problemas tienen un nivel individual (o familiar), sus abordajes deben ir en ese nivel.

La segunda reflexión que se trae a colación y que se vincula con la anterior es que aun cuando en las ideas de las autoridades sobre las situaciones sociales que afectan a los jóvenes de los sectores populares, aparecen reflejadas ciertas racionalidades de corte social que reconocen precisamente las situaciones de este tipo como alentadoras de conductas antisociales, en ellas se recuperan cuestiones sobre la familia y las relaciones de pares, que remiten a contextos inmediatos y no a cuestiones estructurales. Desde la postura de las autoridades, ello parece no representar ningún problema pues efectivamente ambas cuestiones influyen en las conductas antisociales de varios jóvenes, pero para nosotros sí, en tanto termina siendo una postura conservadora que desconoce otro tipo de cuestiones que también abonan a ello y en donde el Estado tendría cierta responsabilidad. Si lo anterior se considera en clave de gobierno, cuando se destacan que las conductas antisociales derivan de cuestiones sociales como las situaciones familiares y las relaciones de pares, se tiende a patologizar el fenómeno como si fuera un problema de ciertos sujetos, lo cual evidentemente no es así, pues su emergencia también está vinculada a otras cuestiones fuera de los círculos concéntricos inmediatos de los jóvenes.

La tercera reflexión es que en las acciones manejadas o promovidas por las autoridades de las preceptorías para contrarrestar o evitar la emergencia de conductas antisociales, se dejan entrever nuevas racionalidades de gobierno que apuntan al control de los jóvenes desde sus conciencias, la gestión de su tiempo y su diversión; sin embargo, se llegan a percibir ciertas posturas que no consideran a las personas, como por ejemplo, no tener espacio para sus opiniones. Ello, desde nuestro punto de vista, es resultado de una suerte de mixtura o cohabitación de doctrinas comunes en las instituciones de control, a saber, de la doctrina de protección integral con la doctrina de la situación irregular, cuyas posturas apelan paradójicamente a reconocer los derechos de los sujetos de atención y a tutelarlos, respectivamente (García, 1994). Esta mixtura no solo pone en tela de juicio los avances reales de las preceptorías en términos de atención a los jóvenes, sino también las posturas precipitadas que ven en ellas ejemplos de instituciones de vanguardia. Como se sabe, la primera doctrina alienta la valoración de los derechos de los niños y jóvenes y su abordaje de forma "aterciopelada" o "suave" para no atentar en contra de dichos derechos, en cambio, la segunda promueve el abordaje de estos sujetos de manera jerárquica, por considerar que no son "adultos" o están en proceso de formación (García, 1994). Si bien, la primera doctrina es la reconocida en la actualidad, la segunda es todavía vigente en gran parte del país, por lo que al cohabitar se crean las bases para que las autoridades de las preceptorías, al mismo que piensan en el control de los niños y jóvenes desde sus conciencias o su diversión para respetarlos, desconsideren sus puntos de vista. 


\section{REFERENCIAS}

Azaola, E. (1991). La institución correccional en México. Una mirada extraviada. México: Siglo xxi Editores.

De Marinis, P. (1999). Gobierno, gubernamentalidad, Foucault y los anglofoucaulteanos. En F. García y R. Ramos (comps.), Globalización, riesgo, reflexividad. Tres temas de la teoría social contemporánea. Madrid, España: Centro de Investigaciones Sociológicas.

Dean, M. (1999). Governmetality. Power and rule in modern society. London: SAGE.

Del Castillo, A. (2006). Conceptos, imágenes y representaciones de la niñez en la ciudad de México, 1880-1920. México: El Colegio de México/Instituto de Investigaciones Dr. José María Luis Mora.

Douglas, M. (1989). Cómo piensan las instituciones. Madrid, España: Alianza Universidad.

Fletes, R. (1996). La infancia abandonada. México: El Colegio de Jalisco.

Funciones ejecutivas y conducta antisocial (s.f.) Recuperado de tesis.uson.mx/digital/ tesis/docs/6391/Capitulo1.pdf

Gajardo, M. y Milos, P. (s.f.). Capacitación de jóvenes en situación de pobreza y riesgo de exclusión: el caso de Chile. Recuperado de www.oei.es/historico/etp/ capacitación_jovenes_situacion_pobreza_ chile.pdf

Galtung, J. (s.f.). La violencia: cultural, estructural y directa. Cuadernos de Estrategia, (183), 147-168.

García, E. (1994). Derecho de la infancia-adolescencia en América Latina: de la situación irregular a la protección integral. Bogotá, Colombia: Forum Pacis.

Gaytán, L. (2006). Sociología de la infancia: nuevas perspectivas. Madrid: Síntesis.

Gobierno del Estado de México. (2013). Reglamento de las preceptorías juveniles regionales de reintegración social del Estado de México. Recuperado de http://dgprs.edomex.gob.mx/sites/dgprs. edomex.gob.mx/files/files/Marc\% 20 Juridico/Reglamento\%20Preceptorias.pdf
González, V. (2012). Una solución a la pobreza: el establecimiento de las escuelas de artes $y$ oficios en México durante el siglo xix. El caso jalisciense. HistoriaRelo. Revista de historia regional y local, 8 (4), 145-171.

Gordon, C. (1991). Governmental rationality: an introduction. En C. Gordon y P. Miller (Eds.). The Foucault effect. Studies in govermentality with two lectures by and interview with Michel Foucault (pp. 1-51). Chicago, eua: The University of Chicago Press.

Graizer, O. (2007). Autonomía escolar como tecnología de gobierno, resistencia y autogobierno. Recuperado de http:// www.anpae.org.br/congressos_antigos/ simposio2007/364.pdf

Grinberg, S. M. (2007). Gubernamentalidad: estudios y perspectivas. Revista Argentina de Sociología, 5 (8), 95-110.

Hener, A. y F. Niszt (s.f.). La gestión preventiva del delito en el contexto de las nuevas racionalidades políticas neoliberales. En Actas del vi Congreso Nacional de Sociología Jurídica. Buenos Aires, Argentina. Recuperado de http://webiigg. sociales.uba.ar/pobmigra/archivos/bec.pdf

Herrera, M. L. (2005). El cuerpo de los niños bajo la mirada de las instituciones sociales y médicas en Puebla a finales del siglo xix. En En el umbral de los cuerpos. Estudios de antropología e historia (pp. 209-239). México: El Colegio de Michoacán/Instituto de Ciencias Sociales y Humanidades-Buap.

Jiménez, P. y Durán, L. (2005). Actividad física $y$ deporte en los jóvenes en riesgo: educación en valores. Apuntes Educación física $y$ deportes, (80), 13-19.

Rose, N. (2007). ¿La muerte de lo social? Re-configuración del territorio de gobierno. Revista Argentina de Sociología, 5 (8), 111-150.

Rose, N. y Miller. P. (1992). Political power beyond of State: problematic of government. British Journal od Sociology, 43 (2), 173-205. 
Sánchez, M. E. (2008). Adolescente homicida: un caso para la psiquiatría criminal. En D. Salazar y M. E. Sánchez, (Comps.). En Niños y adolescentes: normas $y$ transgresiones en México, siglos XVII-XX (pp. 103-116). México: INAH.

Strauss, A. y Corbin, J. (2002). Bases de la investigación cualitativa. Técnicas y procedimientos para desarrollar la teoría fundamentada. Antioquia, Colombia: Universidad de Antioquia.

Tello, N. y Garza, C. (junio de 2004). Subculturas juveniles y violencia. Este País, 34-40. Recuperado de http://archivo.estepais. com/inicio/historicos/159/7_encuesta1_ subculturas_tello.pdf

Fecha de ingreso: 07/11/2017 Fecha de aprobación: 20/07/2018 
